\title{
Clinical outcomes of total laryngectomy for laryngeal carcinoma
}

\author{
Bajaj Y, Shayah A, Sethi N, Harris AT, Bhatti I, Awobem A, Loke D, Woodhead CJ \\ Department of ENT, Leeds Teaching Hospitals, Leeds, UK
}

\begin{abstract}
Background: Controversy exists as to the management of advanced laryngeal carcinoma. In general primary radical surgery is favoured.

Objective: The aim of this study was to analyse the clinical outcome of patients having total laryngectomy for cancer of larynx.

Materials and methods: This study was a retrospective case note review and questionnaires were used for evaluating voice handicap. These laryngectomies included in this study were performed by the senior author (CJW) from January 2001 till June 2007 at Leeds General Infirmary, Leeds. Some of the patients had partial or total pharyngectomy in addition to total laryngectomy.

Results: In this study a total of 59 patients were included. Seventeen $(28.8 \%)$ of these patients had preoperative radiotherapy and laryngectomy was performed for residual or recurrent disease. The initial TNM staging of the tumour ranged from $\mathrm{T}_{1} \mathrm{~N}_{0}$ to $\mathrm{T}_{4} \mathrm{~N}_{2 \mathrm{C}}$. Tracheoesophageal puncture for speech prosthesis was done in 48/59 (81.4\%) patients. Post-operative complications were seen in 30.5\% (18/59) patients. In this study group 9 patients $(15.2 \%)$ developed pharyngocutaneous fistulas. For communication 31/51 (60.8\%) patients were using speech valves. In this study $30.4 \%$ had minimal, $26.1 \%$ moderate and the rest $43.4 \%$ feeling severely handicapped with regards to voice use after total laryngectomy. Five year survival after laryngectomy in this study was $65.2 \%$.
\end{abstract}

Conclusion: Long term disease control and survival is achievable with total laryngectomy with or without postoperative radiotherapy with minimal risks in patients with advanced carcinoma of larynx.

Key words: Laryngectomy, Larynx, Carcinoma

$\mathrm{T}$ he treatment of patients with laryngeal carcinoma should be planned to provide optimal survival, free of disease, with maximum functional results. The treatment for $T_{1}$ and $T_{2}$ lesions usually involves radiotherapy or endoscopic surgery with or without laser. Total laryngectomy alone or in conjunction with neck dissections and/or radiotherapy with or without chemotherapy is used for advanced $\mathrm{T}_{3}$ and $\mathrm{T}_{4}$ lesions ${ }^{1}$.

The first laryngectomy was performed by Billroth in 1873 and for much of the $20^{\text {th }}$ century, this procedure has been recognized as the gold standard treatment for advanced cancers of the larynx and hypopharyn $x^{2}$.

The aim of this study was to analyse the clinical outcome of patients having total laryngectomy for cancer of larynx and hypopharynx. In this study we reviewed the complications and survival rates after total laryngectomy.

\section{Materials and methods}

This study was a retrospective case note review and questionnaires were used for evaluating voice handicap. This study was registered with the audit department at Leeds Teaching hospitals. Patients with squamous carcinoma of the larynx or hypopharynx whose surgical treatment involved total laryngectomy under the care of the senior author (CJW) were identified from the database maintained by the coding department. All the patients were seen preoperatively in the head and neck multidisciplinary clinic.

These laryngectomies included in this study were performed by the senior author (CJW) from January 2001 till June 2007 at Leeds Teaching Hospitals, Leeds. Some patients had initial unsuccessful treatment with radiotherapy. Some of the patients had partial or total pharyngectomy in addition to total laryngectomy. The extent of pharyngeal resection was dictated by the site and local extent of the primary cancer. Therapeutic neck dissection was performed at the time of initial

\footnotetext{
Correspondence

Yogesh Bajaj

2, Tall Trees

Leeds

LS17 7WA, UK

E-mail: ybajaj@hotmail.co.uk
} 
surgery in patients with clinical or radiological neck node involvement. In cases with T3/T4 disease with no radiological evidence of neck nodes, selective neck dissection (levels 2-4) was performed electively. Primary tracheo-oesophageal puncture was performed in most of the patients. Postoperative nutrition was maintained by stomagastric or nasogastric tube feeding. A gastrograffin swallow test was performed on $8^{\text {th }}-9^{\text {th }}$ postoperative day and in the absence of a leak, oral feeding was started. Speech valve was inserted at 10 days postoperatively. The patients were sent home after the oral feeding was established, usually at 10-14 days.

Postoperative radiotherapy was given at the primary site and neck based on the clinico-pathological risks (positive or close surgical margins, pT4 disease, multiple lymph node involvement, extracapsular spread or perineural invasion). The patients were regularly reviewed in the head and neck oncology clinic.

The data for this study was collected from the patient's general case notes and nurses/speech therapist records (for speech valves and humidification systems) and voice handicap index (VHI) questionnaire developed and validated by University of Pittsburgh Voice centre was used to assess the voice handicap ${ }^{3}$. This questionnaire has 30 questions with four possible responses to each questionnaire (Handicap: 0-never, 1-almost never, 2-sometimes, 3-almost always, 4-always). The VHI scores range from 0 to 120 . Scores from 0 -30 indicate minimal handicap, 31-60 denotes moderate handicap and 60-120 represents significant handicap because of voice.

\section{Results}

In this study a total of 59 patients were included (total laryngectomy alone-40; total laryngectomy and partial pharyngectomy-16; total laryngectomy and total pharyngectomy-3). There were 48 male $(81.4 \%)$ and 11 female $(18.6 \%)$ patients in the age range of 41 to 82 years at the time of operation. Of these, 43 patients (72.9\%) were living with their family and 16 patients $(27.1 \%)$ were living on their own. As expected a vast majority of these patients $(93.2 \%, 55 / 59)$ were smokers, smoking in the range of 10-30 cigarettes per day.

Seventeen $(28.8 \%)$ of these patients had preoperative radiotherapy and laryngectomy was performed for residual or recurrent disease. The initial TNM staging of the tumourranged from $\mathrm{T}_{1} \mathrm{~N}_{0}$ to $\mathrm{T}_{4} \mathrm{~N}_{2 \mathrm{C}}$. Tracheoesophageal puncture for speech prosthesis was done in 48/59 (81.4\%) patients (45-primary, 3-secondary). Cricopharyngeal myotomy was performed in $11 / 59(18.6 \%)$ patients. Pharyngeal repair was done primarily in 44/59 (74.5\%) patients. For the rest 15 patients $(25.5 \%)$ pharynx was reconstructed using local and distant flaps (pectoralis major flap-8, radial free forearm flap-3, latissimus dorsi flap-1, jejunal free flap-1, anterolateral thigh skin free flap-2). Simultaneous neck dissection was carried out in $44 / 59$ (74.5\%) patients. In this study group 34 (57.6\%) patients underwent radiotherapy post-operatively.

Post-operative complications were seen in $30.5 \%$ $(18 / 59)$ patients. Some patients had more than one complication (Table 1). Most of the patients had minor complications. There were no post operative deaths. Of the seven patients with haematoma formation, only two had to return to theatre for drainage. The patient with wound breakdown had to undergo repair with pectoralis major flap. Both the chyle leaks settled with conservative management. Of the nine patients $(15.2 \%)$ who developed pharyngocutaneous fistulas, six closed with conservative management and the other three had pectoralis major flap reconstruction for fistula closure.

The follow up of these patients varies from one to six years. As per clinical records 43 patients $(72.9 \%)$ were swallowing normally and 13 patients were on soft diet. Only one patient was unable to swallow postoperatively because of a stroke in the postoperative period. Only five patients $(8.5 \%)$ in this series had undergone pharyngeal dilatations (one patient twice). Of these patients 54/59 $(91.5 \%)$ did not have any problems with the stoma. 5 of $59(8.5 \%)$ patients stoma was narrow. Amongst these patients with narrow stoma, only one patient (1.7\%) has had dilatation done and the others are managing reasonably with a stoma button. None of the patients in this study have required revision procedures on the stoma.

In this study group the patients were using different means of communication (information available on 51 patients). For communication $31 / 51(60.8 \%)$ patients were using speech valves, 6/51 (11.7\%) oesophageal speech, 10/51 (19.6\%) electrolarynx, 3/51 (5.9\%) buccal speech and $1 / 51$ (1.9\%) patients was writing to communicate. The communication was subjectively considered to be good or fairly good in 36/38 (94.7\%) patients and poor in $2 / 38(5.3 \%)$ patients. Forty eight patients had speech valves inserted, of which, nine had their valves removed and the other eight were not using it. Of the 39 patients with speech valves 15 had Provox valves and the rest 24 had Blom Singer valves.

The Voice Handicap Index questionnaire was filled up by 23 patients and the scores ranged from 9 (best) to 120 (worst) in this study. They are ranked in table 2. As per the table $30.4 \%(7 / 23)$ patients had minimal handicap, another $26.1 \%(6 / 23)$ feeling moderate degree of handicap and the rest $43.4 \%$ (10/23) feeling severely handicapped with regards to voice use after total laryngectomy. 
Data on humidification was available on 32 patients. Of these, 14/32 (43.7\%) patients were using Heat Moisture Exchanger system, 15/32 (46.8\%) patients were using laryngectomy bibs and $3 / 32$ patients were not using anything for humidification.

Of the 26 patients for whom the data was available 21 patients $(80.7 \%)$ were looking after their stomas independently and the rest $5 / 21(19.3 \%)$ patients were taking help from a family member for stoma care.
Of the 59 patients in this study $43(72.9 \%)$ are still alive and $16(27.1 \%)$ patients were not alive. The cause of death could be found only in seven patients of which four died of causes related to the laryngeal cancer. Five year survival after laryngectomy in this study was $65.2 \%$ (15/23 patients) and four year survival rate was $64.7 \%$ (22 out of 34 patients)

Table 1: Post-operative complications

\begin{tabular}{|l|c|}
\hline Complication & Number (\%) \\
\hline Haematoma & $7(11.8 \%)$ \\
\hline Seroma formation & $1(1.7 \%)$ \\
\hline Wound breakdown & $1(1.7 \%)$ \\
\hline Chyle leak & $2(3.4 \%)$ \\
\hline Adult Respiratory Distress Syndrome & $1(1.7 \%)$ \\
\hline Stroke & $1(1.7 \%)$ \\
\hline Pharyngocutaneous fistula & $9(15.2 \%)$ \\
\hline
\end{tabular}

Table 2: Voice Handicap Index (VHI) Score.

\begin{tabular}{|c|c|}
\hline Patient number & VHI score \\
\hline 1 & 9 \\
\hline 2 & 10 \\
\hline 3 & 18 \\
\hline 4 & 22 \\
\hline 5 & 26 \\
\hline 6 & 27 \\
\hline 7 & 28 \\
\hline 8 & 32 \\
\hline 9 & 45 \\
\hline 10 & 49 \\
\hline 11 & 51 \\
\hline 12 & 52 \\
\hline 13 & 60 \\
\hline 14 & 65 \\
\hline 15 & 74 \\
\hline 16 & 76 \\
\hline 17 & 87 \\
\hline 18 & 89 \\
\hline 19 & 109 \\
\hline 20 & 110 \\
\hline 21 & 110 \\
\hline 22 & 110 \\
\hline 23 & 120 \\
\hline
\end{tabular}




\section{Discussion}

Controversy exists as to the management of advanced laryngeal carcinoma $\left(\mathrm{T}_{3}-\mathrm{T}_{4} \text { tumours }\right)^{4}$. In general primary radical surgery is favoured ${ }^{5,6}$. Certain authors advocate primary radiotherapy with salvage surgery for recurrent disease ${ }^{7,8,9}$. Total laryngectomy with or without neck dissection and postoperative irradiation is the mainstay of treatment in most centres ${ }^{10}$. The loss of a natural speaking voice after laryngectomy is a difficult hardship for patients. Radiotherapy used as a single modality can preserve voice but the voice preservation and improved quality of life come at the expense of an approximate $10 \%$ decrease in long term survival rates $^{10,11}$. Concurrent Chemoradiotherapy and induction chemotherapy followed by radiotherapy for T3/early $\mathrm{T} 4$ has been tried ${ }^{12,13}$ and authors have mentioned better quality of life scores with this treatment as compared to laryngectomy ${ }^{14}$. The patients in the present study form a relatively heterogeneous group, consisting of individuals with cancers of larynx and hypopharynx, most of them previously untreated but some having failed previous radiotherapy. Majority of the patients had post-operative radiotherapy.

The presence of nodal metastases has consistently been demonstrated to be an important survival determinant in patients with head and neck squamous cell carcinomas ${ }^{15}$. The presence of extracapsular spread in the lymph nodes in laryngeal cancer has been reported as the most significant prognostic indicator ${ }^{16,17}$. The adverse prognostic influence of positive surgical margins after resection of head and neck cancers is well described ${ }^{18}$. Perineural and lymphovascular invasion are associated with poor prognosis in head and neck carcinomas ${ }^{19,20}$. This is because both perineural and lymphovascular invasion were associated with higher rate of locoregional recurrence and lymphovascular invasion was associated with higher rate of distant metastases.

At our centre the usual practice is to carry out ipsilateral neck dissection for most patients with T3/4 disease, either for nodes positive or nodes negative necks. Bilateral neck dissection is carried out for bilateral nodal disease. If there is clinical or radiological evidence of subglottic extension, a level 6 neck dissection is performed in addition. Postoperative radiotherapy is always given for T4 disease. If pathology is pT3 and the number of nodes involved is less than two with no extracapsular spread, then radiotherapy is not necessary. On the other hand, pT3 disease with perineural spread and lymphovascular invasion but no positive nodes may still have radiotherapy post-operatively. This is similar to practice at some other centres ${ }^{21,22}$.

Complications after laryngectomy have a significant impact on morbidity causing prolonged hospitalization and increased health care costs. Many factors have been implicated in the development of complications including previous radiotherapy, preoperative tracheostomy, radical neck dissection and extensive surgery and flap reconstruction. In this study complications after total laryngectomy were seen in nearly $30 \%$ patients which is comparable to postoperative complications in $40 \%$ patients in the study by Ganly et $\mathrm{al}^{23}$ and $30 \%$ in another study $^{24}$.

Pharyngocutaneous fistula is usually evident by the fourth to seventh postoperative day. Fistula is usually present at the superior aspect of the suture line of the pharynx, near the tongue base, but may be evident at the tracheostoma ${ }^{24}$. Fistula formation is usually the most frequent complication after total laryngectomy ${ }^{24}$. The incidence of pharygocutaneous fistula in the present study was $15.2 \%$. This is comparable to the rates in other studies (Ganly et al-17\% ${ }^{23}$; Hall et al $-17.7 \%^{2}$, de Zinis et al- $16 \%{ }^{25}$ ).

At Leeds General Infirmary it is standard practice to delay oral feeding for seven to ten days after laryngectomy. This is similar to the practice at certain other centres ${ }^{2}$. Although there are reports ${ }^{26}$ that early feeding does not increase the rate of fistula, it is our preference to get gastrograffin swallow done before starting oral feeding.

The Voice Handicap Index (VHI) is a question and answer tool developed to help patients and clinicians to quantify the amount of disability the disorder is causing. VHI provides information on the severity of one's voice problems in regard to his/her common activities. In this series $43.4 \%$ felt severely handicapped with regards to voice use after total laryngectomy.

Five year overall survival of $65.2 \%$ in this study group is comparable with $67 \%$ survival reported by Hall et $\mathrm{al}^{2}$ and $71 \%$ reported by Coates et $\mathrm{al}^{27}$ while Foote et al reported a 5 year overall survival of $54 \%{ }^{21}$ and Spector et al reported $53.8 \%$ overall 5 year survival rate ${ }^{28}$.

\section{Conclusions}

Long term disease control and survival is achievable with total laryngectomy with or without postoperative radiotherapy with minimal risks in patients with advanced carcinoma of larynx.

\section{References}

1. Johnson JT, Myers EN, Hao S, Wagner RL. Outcome of open surgical therapy for glottic carcinoma. Ann Otol Rhinol Laryngol. 1993;102:752-5.

2. Hall FT, O'Brien CJ, Clifford AR, McNeil EB, Bron L, Jackson MA. Clinical Outcome following Total Laryngectomy for cancer. Aust N Z J Surg. 2003;73:300-5. 
3. Jacobson BH, Johnson A, Grywalski C, Silbergleit A, Jacobson G, Benninger MS, et al. The Voice Handicap Index (VHI): development and validation. Am J Speech-Lang Pathol. 1997; 6:66-70.

4. Croll GA, Gerritsen GJ, Tiwari RM, Snow GB. Primary radiotherapy with surgery in reserve for advanced laryngeal carcinoma results and complications. Eur J Surg Oncol. 1989;15:350-6.

5. DeSanto LW. T3 glottic cancer: options and consequences of the options. Laryngoscope. 1984; 94:1311-5.

6. Decker JW, Price JC, Goldstein JC. Advanced laryngeal cancer. Arch Otolaryngol Head Neck Surg. 1986;112:1163-7.

7. Lederman M. Radiotherapy of cancer of the larynx. J Laryngol Otol. 1970;84:867-96.

8. Hendrickson FR. Radiation therapy treatment of larynx cancers. Cancer. 1985;55:2058-61.

9. Jones AS, Cook JA, Phillips DE, LLuch ES. Treatment of T3 carcinoma of the larynx by surgery or radiotherapy. Clin Otolaryngol. 1992;17:433-6.

10. Snow GB, Gelber RD. Randomized preoperative radiation therapy for patients with carcinoma of the head and neck. Laryngoscope. 1980;90:930-45.

11. MacNeil BJ, Weichselbaum R, Pauker SG. Speech and survival trade-offs between quality and quantity of life in laryngeal cancer. N Engl J Med. 1981;305:982-7.

12. Wirth LJ, Posner MR. Recent advances in combined modality therapy for locally advanced head and neck cancer. Current Cancer Drug Targets. 2007;7:674-80.

13. Dietz A, Keilholz U, Werner J, Hagen R, Flentie M, Iro H. Current role for induction chemotherapy in head and neck tumours. Laryngorhinootologie. 2008;87:237-43.

14. Boscolo-Rizzo P, Maronato F, Marchiori C, Gava A, Da Mosto MC. Long-term quality of life after total laryngectomy and postoperative radiotherapy versus concurrent chemoradiotherapy for laryngeal preservation. Laryngoscope .2008;118:300-6.

15. Bova R, Goh R, Poulson M, Coman WB. Total Pharyngolaryngectomy for squamous cell carcinoma of the hypopharynx: a review. Laryngoscope .2005; 115:864-9.

16. Moor JW, Jose J, Johnston C, Coatesworth AP, MacLennan KA. Upper aerodigestive tract squamous cell carcinoma: distribution of extracapsular spread and soft tissue deposits in the neck. Acta Otolaryngol.(Stockh). 2004;124:97-101.

17. Hirabayashi H, Koshii K, Ohgaki H, Nakasone Y, Fujisawa T, Syouno N, et al. Extracapsular spread of squamous cell carcinoma in neck lymph nodes: prognostic factor of laryngeal cancer. Laryngoscope. 1991;101:502-6.

18. Vandenbrouck C, Eschwege F, De la Rochefordiere A. Squamous cell carcinoma of the pyriform sinus: retrospective study of 351 cases treated at the Institut Gustave Roussy. Head Neck Surg. 1987;10:4-13.

19. Fagan JJ, Collins B, Barnes L. Perineural invasion in squamous cell carcinoma of the head and neck. Arch.Otolaryngol. Head Neck Surg. 1998;124:637-40.

20. Yilmaz T, Hosal AS, Gedikoglu G. Prognostic significance of vascular and perineural invasion in cancer of the larynx. Am J Otolaryngol. 1998;19:83-8.

21. Foote RL, Olsen KD, Buskirk SJ, Stanley RJ, Suman VJ. Laryngectomy alone for T3 glottic cancer. Head Neck. 1994;16:406-12.

22. Watkinson JC, Gaze MN, Wilson JA. Tumours of the larynx. In: Watkinson JC, Gaze MN, Wilson JA, editors. Stell \& Maran's Head \& Neck Surgery. Butter worth: Oxford; 2004. p. 233-73.

23. Ganly I, Patel S, Matsuo J, Singh B, Kraus D, Boyle J, et al. Postoperative complications of salvage total laryngectomy. Cancer. 2005;103:2073-81.

24. Herranz J, Sarandeses A, Fernandez MF, Barro CV, Vidal JM, Gavilan J. Complications after total laryngectomy in non radiated laryngeal and hypopharyngeal carcinomas. Otolaryngol. Head Neck Surg. 2000;122:892-8.

25. de Zinis LOR, Ferrari L, Tomenzoli D, Premoli G, Parrinello G, Nicolai P. Post laryngectomy pharyngocutaneous fistula: Incidence, predisposing factors and therapy. Head Neck. 1999;21(2):131-8.

26. Boyce SE, Meyers AD. Oral feeding after total laryngectomy. Head Neck. 1989;11:269-73.

27. Coates HL, DeSanto L, Devine KD, Elveback LR. Carcinoma of the supraglottic larynx. Arch. Otolaryngol. Head Neck Surg. 1976;102:686-9.

28. Spector JG, Sessions DG, Lenox J, Simpson J. Management of T3N1 Glottic Carcinoma: therapeutic outcomes. Laryngoscope. 2006;116:106-10. 